\title{
Otra forma (distinta) de enseñar Historia del Pensamiento Económico
}

\section{Another (different) way of learning History of Economic Thought}

José ANTONIO MOLINA-TOUCEDO

https://orcid.org/0000-0003-3589-836X

Universidad de Sevilla

Departamento de Análisis Económico y Economía Política

jamolina@us.es

DOI: http://dx.doi.org/10.12795/9788447231003.078

Pp.: 1677-1691 


\section{Introducción}

Esta comunicación tiene como objetivo implantar un plan de mejora e innovación docente en la asignatura Introducción a la Economía (10 Grado de Administración en Empresas de la Facultad de CC.EE y EE. de la Universidad de Sevilla). El objetivo es profundizar en la metodología docente cognitivista y constructivista, aplicando una variación de la metodología docente de la enseñanza inversa, potenciando el trabajo de búsqueda de información y documentación por equipos, para la elaboración de un trabajo y su posterior exposición ante tribunal por todos los componentes de cada equipo. Seguidamente se realizó una actividad de refuerzo del conocimiento, consistente en una actividad de gamificación competitiva por equipos. Se elaboró una encuesta inicial de testeo de conocimientos para averiguar los modelos mentales y de conocimiento de los estudiantes y calibrar el gradiente de dificultad del objeto de estudio. Se finalizó la actividad realizando de nuevo la encuesta inicial. Los resultados obtenidos resultan francamente alentadores.

\section{Descripción del contexto de la asignatura}

Este contenido es parte del Proyecto Docente de la asignatura Introducción a la Economía de 10. Es una asignatura básica del primer cuatrimestre del Grado de Administración de Empresas de la Universidad de Sevilla, que consta de 6 créditos CTS y un total de 9 grupos, 5 de mañana y 4 de tarde. El Grupo M9, grupo objetivo, es un grupo de docencia en inglés de la Facultad de CC.EE. y EE. de Sevilla en GADE en el Curso 2020-21 y consta de un total de 23 alumnos matriculados. El lugar es el Aula 21 de la Facultad de CC.EE. y EE, que cuenta con 23 plazas según las normas sanitarias ANTICOVID 19. El aula está dotada con cañón, Ciclos de Mejora en el Aula (2020). Experiencias de Innovación Docente de la US
Esta obra se distribuye con la licencia Creative Commons 
ordenador y servicio de megafonía. Se ha escogido como objeto de estudio del Ciclo de Mejora en el Aula (CIMA a partir de ahora) el tema del proyecto docente sobre la Historia del Pensamiento Económico (tema 2). La finalidad de la enseñanza es, romper la natural desidia de los estudiantes sobre la historia del pensamiento económico, tratando de animar a los alumnos a indagar sobre las principales ideas y autores que han escrito sobre economía a lo largo del tiempo, haciéndoles reflexionar sobre la conexión entre la realidad social y económica de cada época y los paradigmas del pensamiento económico dominantes en las mismas, de una forma novedosa e interactiva, individual y por equipos (Finkel, 2008).

\section{Diseño previo del CIMA y modelo metodológico posible}

\section{Mapa de contenidos}

La Historia del Pensamiento Económico impartida en esta asignatura de Introducción a la Economía, es muy escueta. El objetivo es trasladar ideas generales, claras y sintéticas sobre la evolución histórica de la humanidad en su forma de pensar y analizar los hechos de contenido económico. Las principales escuelas del pensamiento económico: fisiocrática, clásica, histórica, socialista, comunista, marginalismo, keynesianismo y tendencias actuales, son estudiadas de forma sucinta, centrándose en sus principales autores e ideas fundamentales.

En la figura 1 se presenta el mapa de contenidos conceptuales en forma de árbol de interrelación entre escuelas de Pensamiento Económico.

Ciclos de Mejora en el Aula (2020). Experiencias de Innovación Docente de la US Esta obra se distribuye con la licencia Creative Commons 


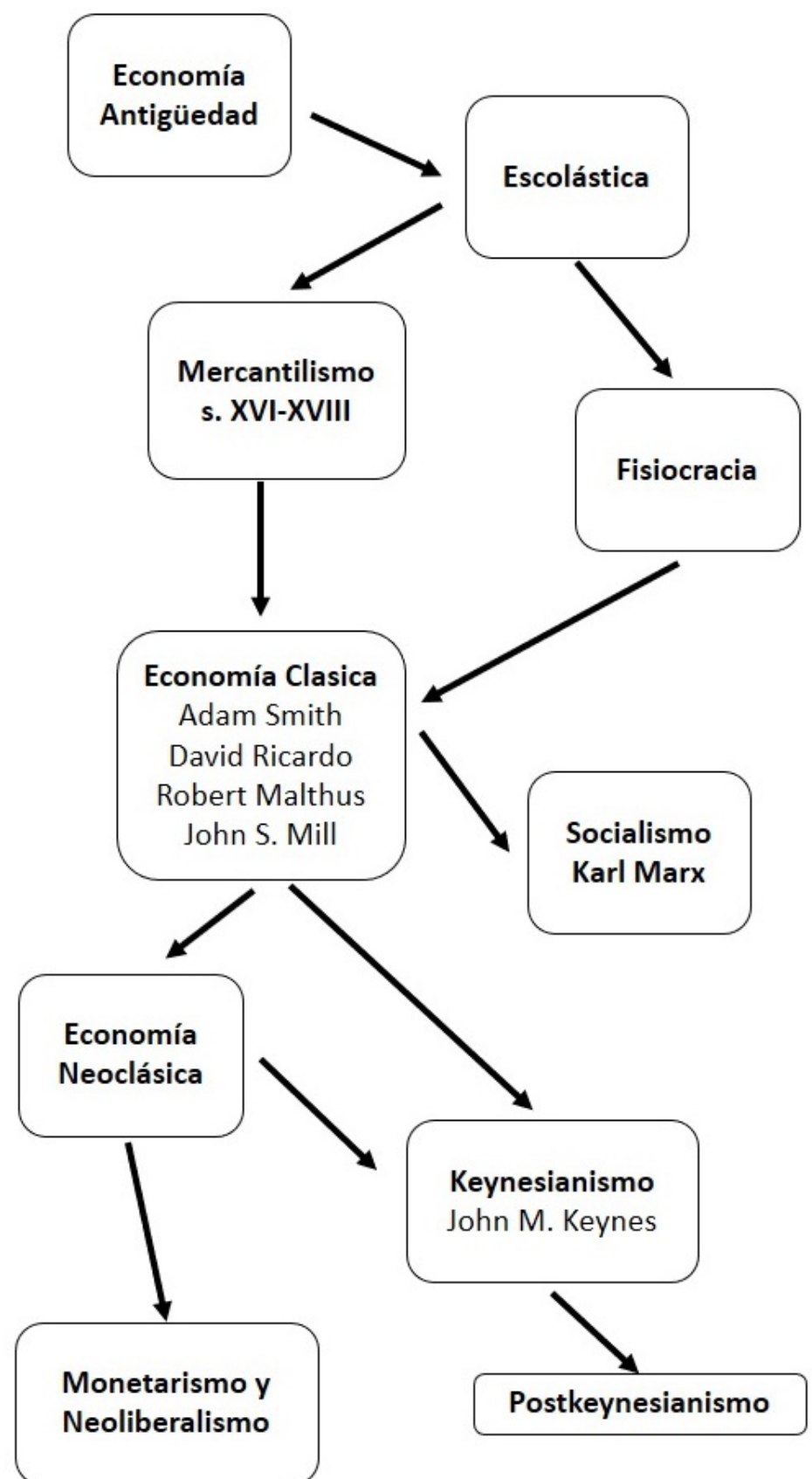

Figura 1. Mapa de contenidos conceptuales

Ciclos de Mejora en el Aula (2020). Experiencias de Innovación Docente de la US 


\section{Modelo metodológico posible}

Se ha utilizado una variación de la metodología docente de la enseñanza inversa, término acuñado por primera vez por Bergmann y Sams: "Es un enfoque pedagógico en el que la instrucción directa se desplaza al espacio del aprendizaje individual; como resultado de ello, el espacio del grupo se transforma en un ambiente de aprendizaje dinámico e interactivo" (Bergmann y Sams, 2012). En concreto, se potenció el trabajo de búsqueda de información y documentación por equipos (en lugar del formato unipersonal). Además se realizó una encuesta inicial individual en el grupo objetivo (M9), con la que se trató de averiguar los conocimientos previos de los alumnos, sobre los principales autores e ideas de las escuelas de pensamiento económico. Seguidamente, en el grupo objetivo (M9) se confeccionaron 6 equipos de trabajo (4-3 miembros cada uno) y se procedió a la explicación del problema ( $\mathrm{Pr}$ ) de cada equipo: la elaboración de un trabajo escrito y del material de cada uno de los 6 epígrafes del tema que se asignaron por sorteo. En dicha sesión se proporcionó la Bibliografía de apoyo necesaria para la elaboración, formas de acceder a ella, así como la estructura, normas de estilo y extensión del trabajo y de la presentación. En la siguiente sesión se trabajó por equipos en la elaboración del trabajo dentro de la clase, tomando conciencia los alumnos de su comprensión de sus ideas respecto al problema (le). Actuando el docente como tutor en la resolución de dudas y problemas (Porlan et al., 2017).

Seguidamente cada equipo de trabajo realizó una actividad de contraste $\left(\mathrm{AC}_{1}\right)$, presentando su trabajo al resto de compañeros y a un tribunal de 2 profesores de la asignatura, estableciéndose un turno de palabra de $10 \mathrm{~m}$. por equipo y un turno de preguntas de los compañeros y del tribunal de $5 \mathrm{~m}$. En una última sesión del grupo objetivo (M9), se realizó una segunda actividad de contraste $\left(A_{2}\right)$; un ejercicio competitivo de gamificación, a través de la herramienta Kahoot en su modalidad de juegos por equipos.

Ciclos de Mejora en el Aula (2020). Experiencias de Innovación Docente de la US Esta obra se distribuye con la licencia Creative Commons 
Se elaboró una batería de preguntas estipulándose un periodo de respuesta de 3-4 minutos, que permitió el diálogo y el consenso en cada equipo antes de responder. Finalmente, se volvió a pasar la Encuesta Inicial, con la opción de preguntas y respuestas en orden aleatorio. Los resultados permitieron comparar los niveles de conocimiento en las fases inicial y final, lo que posibilitó evaluar el aprendizaje alcanzado y obtener conclusiones (C) (Porlan et al.,2017).

Con toda esta metodología docente se ha tratado de recrear los 5 elementos del Aprendizaje Crítico Natural: problema intrigante (búsqueda de información), orientación del docente (aporte de bibliografía y tutorización del proceso), actividad intelectual (elaboración del trabajo y de la presentación del mismo), autorespuesta del alumno (interiorización de la escuela de pensamiento y redacción del trabajo) y conclusiones de final de actividad (Bain,

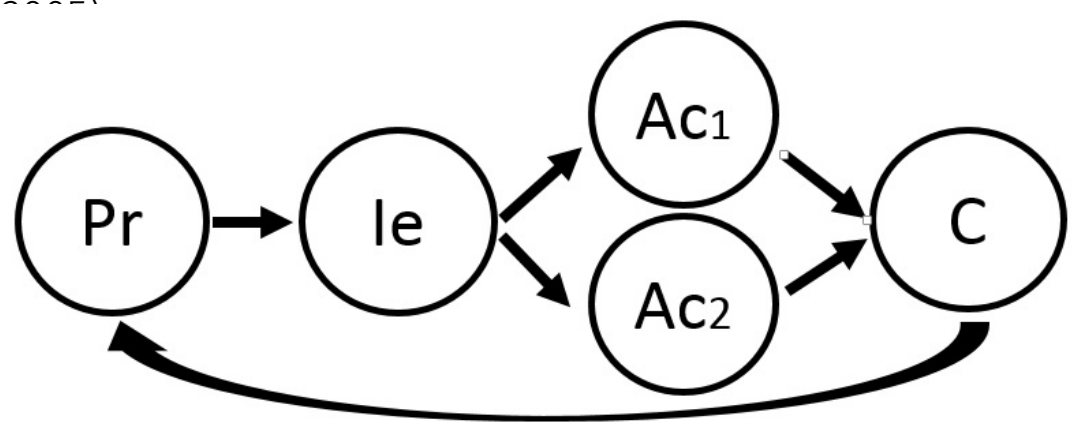

Figura 2. Modelo metodológico posible aplicado

\section{Aplicación del CIMA}

La actividad se desarrolló en un total de 4 sesiones de 2 horas cada una de ellas, los días 19,21,26 y 28 de Octubre de 2020. Dado que es un grupo en inglés, todas las actividades se realizaron en este idioma. Además se cumplió la normativa de seguridad sanitaria ANTICOVID19 elaborada por el SEPRUS (Servicio Prevención Universidad de Sevilla)

Ciclos de Mejora en el Aula (2020). Experiencias de Innovación Docente de la US Esta obra se distribuye con la licencia Creative Commons 
aplicable a las clases presenciales. Dado el reducido número de alumnos (23) fue factible iniciar las clases en el Escenario 0. Se cumplió el siguiente guion:

Sesión 1ạ (120 minutos)

Se les presentó el CIMA, y se realizó la encuesta inicial individual, compuesta por un total de 20 preguntas. Dichas preguntas versaron sobre los principales autores y escuelas de Pensamiento Económico. Para la ejecución de la encuesta se utilizó la herramienta de gamificación Kahoot, en su modalidad de encuesta (survey) y en el modo de respuesta individual. Por si algún alumno no estuviese habituado a esta herramienta se realizará un primer juego de presentación en modalidad quizz.

En la figura 3 se refleja la captura de pantalla con los resultados de la encuesta inicial individual y en las figura 4 y 5 , el detalle de los resultados de aciertos por pregunta. Los bajos niveles de respuesta correctas son completamente normales dado que es un materia aún no impartida y de la que los alumnos suelen tener un escaso conocimiento previo.

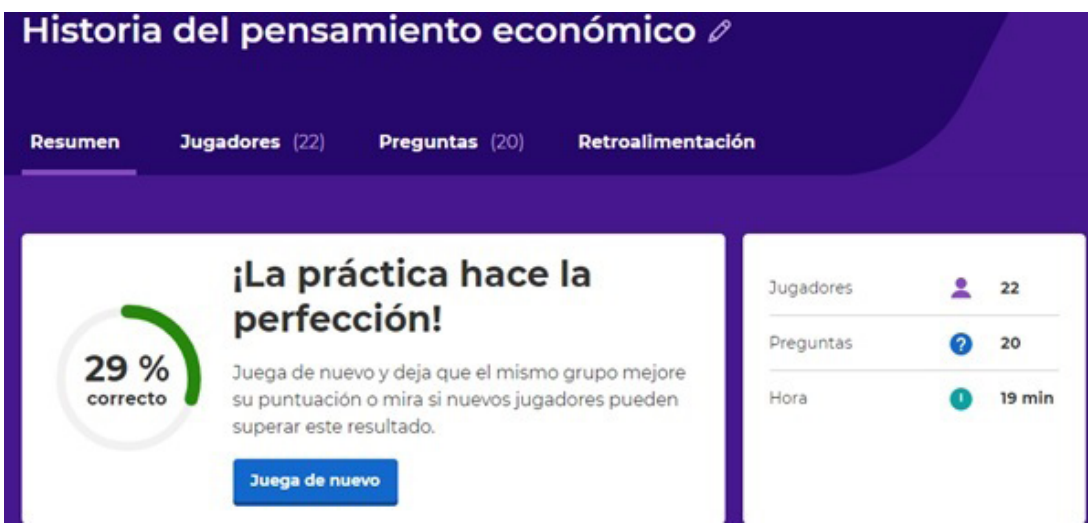

Figura 3. Resumen Cuestionario Inicial con \% respuestas correctas

Ciclos de Mejora en el Aula (2020). Experiencias de Innovación Docente de la US Esta obra se distribuye con la licencia Creative Commons Reconocimiento-NoComercial-SinObraDerivada Internacional (CC BY-NC-ND 4.0.) 


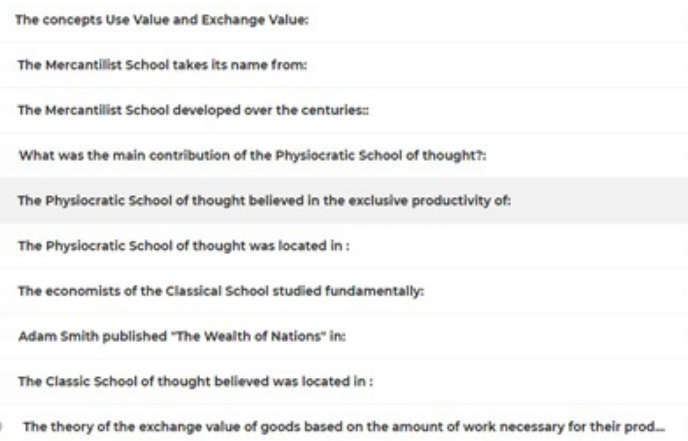

Figura 4. Detalle preguntas 1 a 10 Cuestionario Inicial con \% respuestas correctas
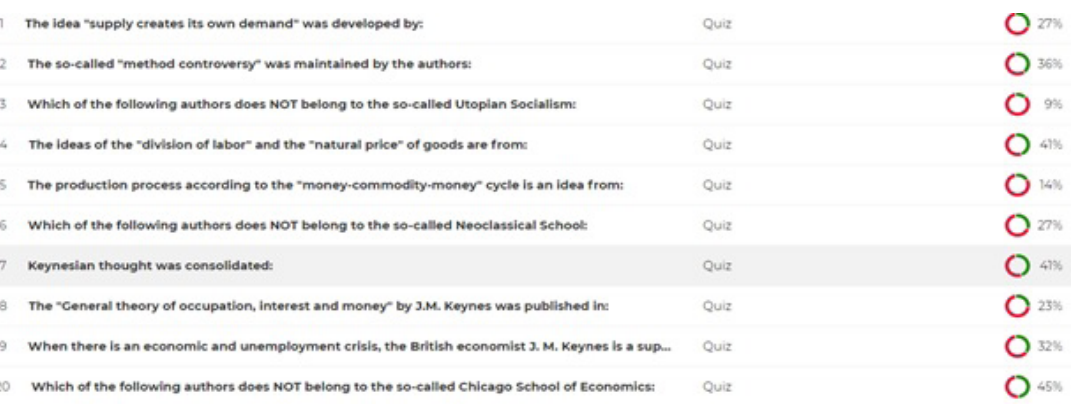

Figura 5. Detalle preguntas 11 a 20 Cuestionario Inicial con \% respuestas correctas

Seguidamente se confeccionaron aleatoriamente 6 equipos de trabajo (4-3 miembros cada uno) y se procedió a la explicación del objetivo de cada equipo: la elaboración de un trabajo escrito y del material para su exposición en clase, de cada uno de los 6 epígrafes del tema 2 (que se asignaron también por sorteo). Se aportó y explicó la bibliografía de apoyo elegida, la forma de documentarse, así como la extensión y normas de estilo del trabajo y de su presentación.

Durante esta sesión se respiró un ambiente muy relajado. Los alumnos disfrutaron de la sesión. Se divirtieron con los Kahoots, viviendo a tope la competitividad de los

Ciclos de Mejora en el Aula (2020). Experiencias de Innovación Docente de la US Esta obra se distribuye con la licencia Creative Commons 
puntos que esta herramienta recrea y que teatraliza con la elaboración de un pódium final, con los tres participantes con mayor puntuación.

\section{Sesión 2ạ (120 minutos)}

En esta sesión se trabajó por equipos en la elaboración del trabajo y de su exposición. El docente actuó de tutor de dicha sesión, apoyando y resolviendo dudas y consultas de los diferentes equipos de trabajo. Los equipos comenzaron a conocerse y a dividirse el trabajo. El docente fue pasando por los 6 equipos, oyendo sus diálogos y aportando apoyo según la necesidad detectada en cada equipo, adaptándose al nivel de cada equipo de alumnos, con una construcción progresiva de los conocimientos (hipótesis de progresión) (García, 1998). Las medidas ANTICOVID19 obligaron a mantener separación física entre los componentes de los grupos, lo que entorpeció algo la comunicación entre ellos y por tanto la dinámica.

\section{Sesión 3ạ (120 minutos)}

Con esta sesión arrancamos la "Semana del Pensamiento Económico". En ella cada equipo presentó su trabajo al resto de compañeros y a un tribunal de 2 profesores de la asignatura. Todos los equipos supieron gestionar bien el escaso tiempo de presentación (10 m.). La falta de dominio del idioma inglés resulto un hándicap en la presentación de algunos alumnos, al mermar la capacidad oratoria de los mismos. El turno de preguntas posterior a la presentación de cada equipo, aunque estuvo relativamente animado, a juicio de los docentes precisaría incentivar la participación de los alumnos de alguna forma.

En la rúbrica de evaluación del tribunal se consideraron los siguiente ítems: material del trabajo aportado por el equipo, material de la presentación elaborada, intervención personal durante la presentación, evaluación por pares remitida de forma anónima a cada componente, y

Ciclos de Mejora en el Aula (2020). Experiencias de Innovación Docente de la US Esta obra se distribuye con la licencia Creative Commons 
resolución de las dudas y preguntas que se le plantearon en el turno de debate post exposición.

\section{Sesión 4ạ (120 minutos)}

En la última sesión (final de la Semana del Pensamiento Económico), se realizó una actividad competitiva de gamificación por equipos, para reforzar las ideas principales de las diferentes escuelas de pensamiento económico. Utilizando la herramienta Kahoot, en su modalidad de juegos por equipos, se creó una batería de preguntas (15), diferentes a las de la encuesta inicial, estipulándose un periodo de respuesta de 3-4 minutos, lo cual potenció el diálogo y el consenso previo en cada equipo antes de emitir sus respuestas, en donde la celeridad de la misma, contó con un bonus positivo en la puntuación. Se produjeron escenas de auténtica tensión en aquellos momentos en los que la respuesta no estaba clara y se producían acalorados debates para acordar la respuesta. En las figuras 6 y 7 , se muestran el extracto de los resultados globales y por equipos de esta encuesta, en donde se aprecia un significativo avance respecto a la encuesta inicial.

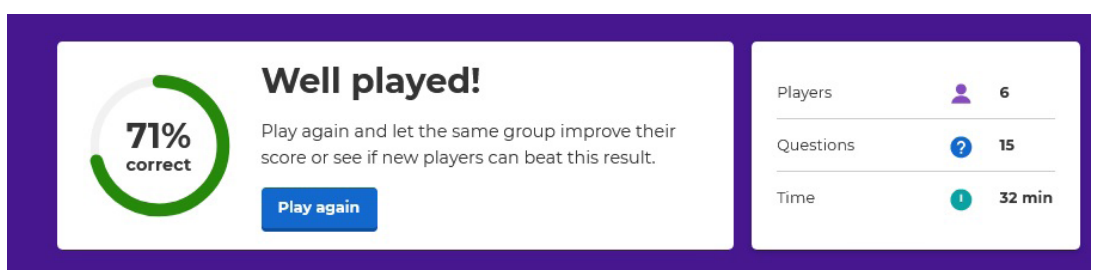

Figura 6. Resumen Cuestionario por equipos con \% respuestas correctas

\begin{tabular}{|c|c|c|}
\hline All (6) & Didn't finish (5) & \\
\hline Nickname $\vee$ & Rank $\vee$ & Correct answers $\vee$ \\
\hline team 4 & 1 & $80 \%$ \\
\hline Team 2 & 2 & $87 \%$ \\
\hline Team 6 & 3 & O $80 \%$ \\
\hline team 5 & 4 & O ${ }_{67 \%}$ \\
\hline Team 3 & 5 & O $60 \%$ \\
\hline Team 1 & 6 & ( ${ }_{53} \%$ \\
\hline
\end{tabular}

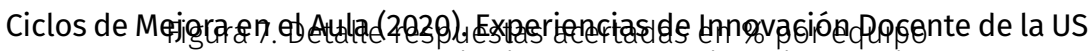
Esta obra se distribuye con la licencia Creative Commons Reconocimiento-NoComercial-SinObraDerivada Internacional (CC BY-NC-ND 4.0.) 
Finalmente se les volvió a pasar la Encuesta Inicial, con la opción de preguntas y respuestas en orden aleatorio (para evitar el efecto memoria respecto a la encuesta inicial). En las figuras 8, 9 y 10, se representan los resultados alcanzados, que muestran un significativo y positivo avance comparados con los obtenidos en la encuesta inicial.

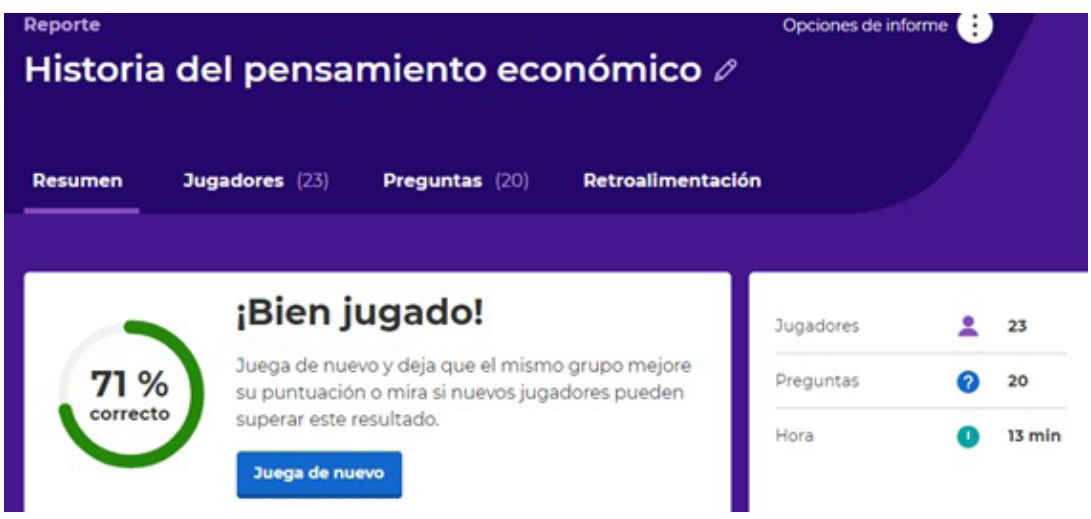

Figura 8. Resumen Cuestionario Final con \% respuestas correctas
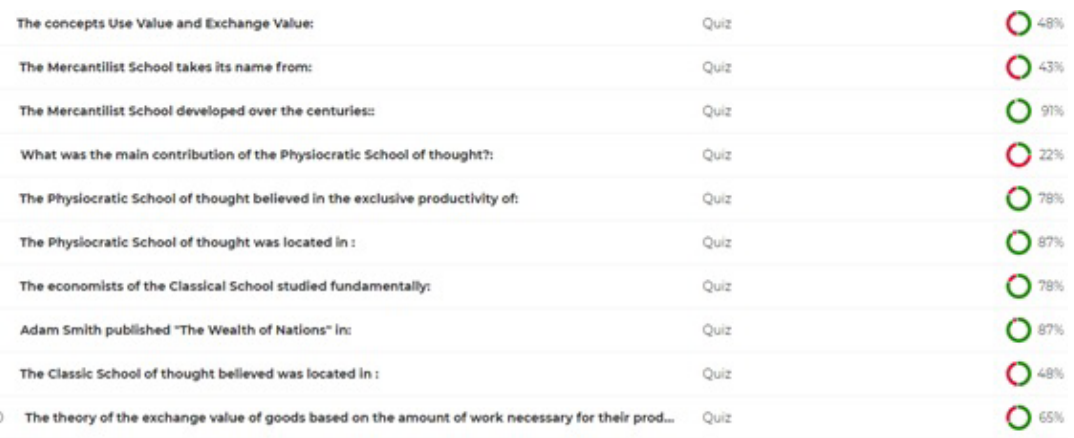

Figura 9. Detalle preguntas 1 a 10 Cuestionario Final con \% respuestas correctas

Ciclos de Mejora en el Aula (2020). Experiencias de Innovación Docente de la US (c) Esta obra se distribuye con la licencia Creative Commons Reconocimiento-NoComercial-SinObraDerivada 
Figura 10. Detalle preguntas 11 a 20 Cuestionario Final con \% respuestas correctas

Tabla 1. Extracto secuencia actividades

\begin{tabular}{|c|c|c|c|}
\hline SESIÓN & TIPO ACTIVIDAD & $\begin{array}{l}\text { DESCRIPCIÓN Y } \\
\text { RECURSOS }\end{array}$ & TIEMPO \\
\hline $\begin{array}{l}\text { Primera } \\
(19 / 10)\end{array}$ & $\begin{array}{l}\text { Presentación. Encuesta } \\
\text { Individual (Kahoot) con } \\
\text { preguntas sobre autores y } \\
\text { escuelas del Pensamiento } \\
\text { Económico. }\end{array}$ & $\begin{array}{l}\text { En esta sesión se realizó } \\
\text { la Encuesta Inicial } \\
\text { individual. Compuesta } \\
\text { por } 20 \text { preguntas. A } \\
\text { continuación elección } \\
\text { aleatoria de los } 6 \text { equipos } \\
\text { de trabajo. Explicación de } \\
\text { la bibliografía de apoyo, } \\
\text { forma de documentación, } \\
\text { extensión y normas de } \\
\text { estilo del trabajo y de su } \\
\text { presentación. }\end{array}$ & $120 \mathrm{~m}$. \\
\hline $\begin{array}{l}\text { Segunda } \\
(21 / 10)\end{array}$ & $\begin{array}{l}\text { Tutorización trabajo por } \\
\text { equipos en clase. }\end{array}$ & $\begin{array}{l}\text { El docente tutoriza } \\
\text { el trabajo en equipo. } \\
\text { Permanece de forma } \\
\text { estática como oyente } \\
\text { durante un tiempo en } \\
\text { cada equipo de trabajo y } \\
\text { resuelve de forma activa } \\
\text { las dudas y cuestiones } \\
\text { de procedimiento que } \\
\text { planteen los equipos. }\end{array}$ & $120 \mathrm{~m}$. \\
\hline
\end{tabular}

Ciclos de Mejora en el Aula (2020). Experiencias de Innovación Docente de la US Esta obra se distribuye con la licencia Creative Commons 


\begin{tabular}{|c|c|c|c|}
\hline $\begin{array}{l}\text { Tercera } \\
(26 / 10)\end{array}$ & $\begin{array}{l}\text { Presentación de los } \\
\text { trabajos por equipos }\end{array}$ & $\begin{array}{l}\text { Cada equipo contará con } \\
10 \mathrm{~m} \text {. para su presentación. } \\
\text { Será obligatoria la } \\
\text { participación de todos los } \\
\text { miembros del equipo. Se } \\
\text { establecerá un turno de } \\
\text { preguntas de compañeros } \\
\text { y miembros del Tribunal } \\
\text { de } 5 \mathrm{~m} \text {. }\end{array}$ & $120 \mathrm{~m}$. \\
\hline $\begin{array}{l}\text { Cuarta } \\
(28 / 10)\end{array}$ & $\begin{array}{lr}\text { Campeonato } & \text { de } \\
\text { Pensamiento } & \text { Económico } \\
\text { por equipos. } & \end{array}$ & $\begin{array}{l}\text { Competición por equipos } \\
\text { de trabajo consistente } \\
\text { en 10-15 preguntas en la } \\
\text { modalidad de equipos } \\
\text { (quizz team mode). } \\
\text { Repetición de la Encuesta } \\
\text { Inicial individual. } \\
\text { Evaluación, comentarios y } \\
\text { aportaciones }\end{array}$ & $120 \mathrm{~m}$. \\
\hline
\end{tabular}

\section{Evaluación. Conclusiones y discusión}

Podemos afirmar que la aplicación del Ciclo de Mejora ha permitido romper el rechazo natural que los alumnos suelen mostrar hacia la materia de la Historia del Pensamiento Económico. La utilización de la herramienta de la educación inversa permitió trasladar la dinámica del aprendizaje al propio alumno a través de equipos de trabajo tutorizados. La metodología del trabajo en equipo y de la presentación de los trabajos antes tribunal, permitieron trabajar competencias transversales y complementarias a la absorción de conocimientos, tales como la interacción entre pares para la resolución de un problema, la redacción de documentos en formato científico, la preparación de presentaciones en público y la ejecución de una exposición oral ante un auditorio de oyentes y con evaluadores.

En la tabla 2 se muestran los datos comparados de los test inicial y final, consistentes en 20 preguntas tipo test, de 4 opciones y con una sola correcta (ver en Anexo 1 detalle, contenido de las preguntas y datos en test inicial y final). Los resultados fueron francamente positivos y alentadores.

Ciclos de Mejora en el Aula (2020). Experiencias de Innovación Docente de la US Esta obra se distribuye con la licencia Creative Commons 
Tabla 2. Datos Encuesta Inicial y Final

\begin{tabular}{|c|c|c|c|}
\cline { 3 - 4 } \multicolumn{1}{c|}{} & $\begin{array}{c}\text { TEST INICIAL } \\
14-10-20\end{array}$ & $\begin{array}{c}\text { TEST FINAL. } \\
28-10-20\end{array}$ & $\begin{array}{c}\text { TEST FINAL/ } \\
\text { TEST INICIAL } \\
(\%)\end{array}$ \\
\hline NoALUMNOS-AS & 19 & 22 & $\mathbf{2 5 9 \%}$ \\
\cline { 1 - 1 } $\begin{array}{c}\text { MEDIA No PUNTOS/ALUMNO-A } \\
\text { MEDIA No RESPUESTA CORRECTAS } \\
\text { (TOTAL 20)/ALUMNO-A }\end{array}$ & 4.723 & 12.222 & $\mathbf{2 3 6 \%}$ \\
\hline
\end{tabular}

Entre los cambios y mejoras a introducir para futuros cursos tenemos los siguientes:

- Aportar más bases bibliográficas en español para la búsqueda del material de los trabajos por equipos

- Diseñar y aplicar una evaluación por pares que permita tener información sobre el nivel de implicación y de trabajo dentro de los grupos.

- Diseñar y aplicar un sistema para propiciar e incentivar la fase de preguntas de los demás alumnos de después de la presentación de cada grupo.

Ciclos de Mejora en el Aula (2020). Experiencias de Innovación Docente de la US Esta obra se distribuye con la licencia Creative Commons 
Palabras clave: Introducción a la economía, grado en economía, docencia universitaria, experimentación docente universitaria, historia del pensamiento económico, educación inversa

Keywords: Introduction to economics, degree in economics, university teaching, university teaching experimentation, history of economic thought, flipped classroom.

\section{Referencias}

Bain, K. (2005). Lo que hacen los mejores profesores universitarios. Valencia: Servicio de Publicaciones de la Universidad de Valencia

Bergmann, J. y Sams, A. (2012). Flip your classroom: Reach every student in every class every day. International society for technology in education.

Finkel, D. (2008). Dar clase con la boca cerrada. Valencia: Servicio de Publicaciones de la Universidad de Valencia

García, J. E. (1998). Hacia una teoría alternativa sobre los contenidos escolares. Sevilla: Diada.

Porlán, R. (Coord.) (2017). Enseñanza universitaria. Cómo mejorarla. Madrid: Morata.

Ciclos de Mejora en el Aula (2020). Experiencias de Innovación Docente de la US Esta obra se distribuye con la licencia Creative Commons 